\title{
Disease Object
}

National Cancer Institute

\section{Source}

National Cancer Institute. Disease Object. NCI Thesaurus. Code C43571.

Disease objects specify a disease name and ID; disease objects also provide access to:

ontological relations to other diseases; clinical trial protocols treating the disease; and specific histologies associated with instances of the disease. (caBIO) 